\title{
P0546 - HD-6277, a novel GPR40 agonist, improves glycemic control in type 2 diabetic rodents with beta- cell secondary failure
}

\author{
Author: G. Yang, Y.-S. Kim, C. Kim, S. Lee, H.-S. Choi, S. Kang, D. Kim, H. Yu, J. Rhee, \\ D. Kim;
} New Drug Discovery Lab., Hyundai Pharm, Yongin-si, Korea, Republic of.

\section{Background and aims:}

Insulin secretagogues and therapeutics for type 2 diabetic (T2D) mellitus cause adverse effects including hypoglycemia, pancreatic beta-cell secondary failure and decrease of beta-cell mass. In addition, T2D typically requires long-term administration of the drugs. Long-term administration of sulfonylurea causes the reduction in the efficacy, which is called 'secondary failure' and considered a major clinical difficulty as well as side effect. Therefore, there is a need for a new drug that glucose-dependent insulin secretion with low risk of adverse effects associated with sulfonylurea treatment. Recently, reports demonstrated that G-proteincoupled receptor 40 (GPR40) agonist can improve glycemic control by glucose-dependent insulin secretions. Goto-Kakizaki (GK) rats have known as type 2 diabetic model with secondary failure and apoptosis of pancreatic beta-cell. In this study, we evaluated the efficacy of HD-6277 in T2D rodent models with secondary beta-cell failure.

\section{Materials and methods:}

in vitro: HD-6277 was evaluated an EC50 value in GPR40-transient expressed CHO cell line. And glucosestimulated insulin (GSIS) was tested in INS-1.

in vivo: HD-6277 and glimepiride were administered to GK rats of 8 (young) and 26 (old) weeks old, respectively. And glucose tolerance tests (GTT) were performed at the starting and the ending point of the chronic administration.

\section{Results:}

HD-6277 showed an EC50 value of $15 \mathrm{nM}$ in GPR40-transient expressed CHO cell line, and increased GSIS in INS-1 cells. Administration of HD-6277 and glimepiride improved glycemic control and insulin secretion, compared to vehicle control groups in young GK rats, however, chronic administration of glimepiride cause loss of glycemic control efficacy, whereas HD-6277 did not. At the end of chronic administration, glimepiridetreated group showed lower glycemic control and insulin secretion during GTT than HD-6277-treated group. In administration of HD-6277 and glimepiride to old GK rats, HD-6277-treated group showed higher glycemic control than vehicle control groups, however glimepiride-treated group did not.

Conclusion: In young GK rats which may have lower beta-cell failure, HD-6277 and glimepiride showed enhanced glycemic control. However, after progression of beta-cell failure by repeated glimepirideadministration and aging, glimepiride-treated group showed impaired glycemic control. However, HD-6277 maintained beta-cell function and glycemic control efficacy against to beta-cell secondary failure. Taken together, we propose that HD-6277 can be a potent agent for type 2 diabetic patients with beta-cell secondary failure.
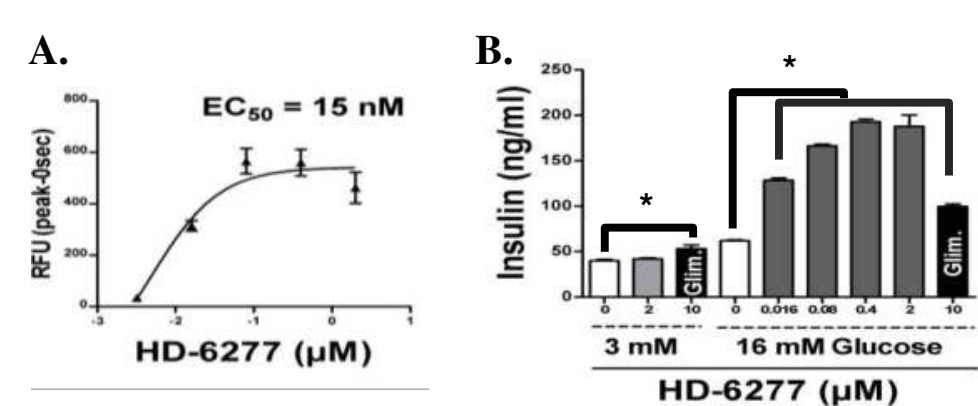

Fig.1 Pharmacological Properties of HD-6277

(A) $\mathrm{EC}_{50}$ value in GPR40-transient expressed $\mathrm{CHO}$ cell line. (B) Glucosestimulated insulin secretion (GSIS) test in INS-1 Pancreatic beta-cells; HD$6277(0.016 \sim 2 \mu \mathrm{M})$, Glimepiride (Glim.;10 $\mu \mathrm{M})$ for $1 \mathrm{~h} .{ }^{*} p<0.05$ : Student t-test.
Reference:

- Mol Cell Endocrinol. 2009 Jan 15;297(1-2):73-85.

- J Pharmacokinet Pharmacodyn. 2011 Feb;38(1):143-62.

- J Clin Invest. 2006 Jul;116(7):1802-12.

Acknowledgements: This research was supported by a grant of the Korea Health Technology R\&D Project through the Korea Health Industry Development Institute (KHIDI), funded by the Ministry of Health \& Welfare, Republic of Korea (grant number : HI14C2710).

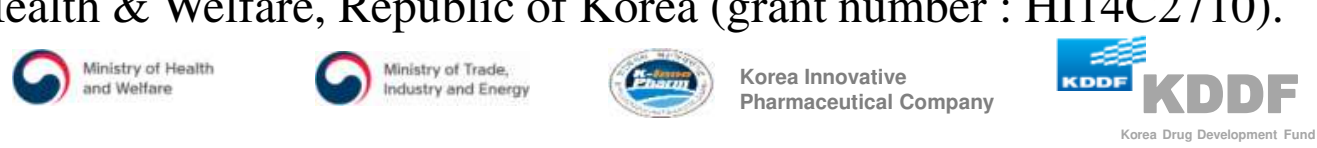

